\title{
GENERALIZED FRACTIONAL HERMITE-HADAMARD INEQUALITIES
}

\author{
MUHAMMAD ASLAM NOOR, KHALIDA INAYAT NOOR, \\ AND MUHAMMAD UZAIR AWAN
}

Received 25 February, 2014

\begin{abstract}
In this paper, we derive some Hermite-Hadamard type inequalities via $s$-convex functions of first and second sense respectively. These inequalities involve $k$-Riemann-Liouville fractional integrals. We also discuss some special cases.
\end{abstract}

2010 Mathematics Subject Classification: 26A33; 26D15; 26A51

Keywords: convex, $s$-convex functions, Hermite-Hadamard inequality, $k$-Riemann-Liouville fractional integrals

\section{INTRODUCTION}

Theory of convexity has many applications in different fields of pure and applied sciences. Due to this many researchers have investigated it in different directions. Resultantly the concept of convexity has been extended and generalized in numerous ways using novel and innovative ideas, see [1-9, 18].

Theory of convexity also plays significant role in mathematical inequalities. One of the most famous inequality which provides necessary and sufficient condition for a function to be convex is Hermite-Hadamard's inequality. For some recent investigations on Hermite-Hadamard type inequalities interested readers are referred to [4, 6-8, 11-17, 19-24].

Recently Sarikaya et al. [16] has introduced the notion of $k$-Riemann-Liouville fractional integrals and discussed some of its interesting properties. It is worth to mention here that $k$-Riemann-Liouville fractional integral is the generalized form of classical Riemann-Liouville fractional integrals as when $k \rightarrow 1$ we get the classical RiemannLiouville fractional.

In this paper, we consider the $k$-Riemann-Liouville fractional integrals and derive several new Hermite-Hadamard type inequalities via $s$-convex functions of first and second sense which involve $k$-Riemann-Liouville fractional integrals. The ideas and techniques of this paper may inspire interested readers to explore applications and 
other aspects of Hermite-Hadamard inequalities in other fields of pure and applied sciences. This is the main motivation of this paper.

\section{PRELIMINARIES}

In this section we recall some previously known concepts. First of all let $I=$ $[a, b] \subseteq \mathbb{R}$ be the interval and $\mathbb{R}$ be the set of real numbers.

Let us suppose that $s \in[0,1]$.

Definition 1 ([18]). A function $f: I \rightarrow \mathbb{R}$ is said to be a $s$-convex function in the first sense, if

$$
f(t x+(1-t) y) \leq t^{s} f(x)+\left(1-t^{s}\right) f(y), \quad \forall x, y \in I, t \in[0,1] .
$$

Definition 2 ([1]). A function $f: I \rightarrow \mathbb{R}$ is said to be a $s$-convex function in the second sense (also called Breckner convex), if

$$
f(t x+(1-t) y) \leq t^{s} f(x)+(1-t)^{s} f(y), \quad \forall x, y \in I, t \in[0,1] .
$$

Now, we recall the concept of $k$-Riemann-Liouville fractional integrals. Let $f$ be piecewise continuous on $I^{*}=(0, \infty)$ and integrable on any finite subinterval of $I=[0, \infty]$. Then for $t>0$, we consider $k$-Riemann-Liouville fractional integral of $f$ of order $\alpha$

$$
{ }_{k} J_{a}^{\alpha} f(x)=\frac{1}{k \Gamma_{k}(\alpha)} \int_{a}^{x}(x-t)^{\frac{\alpha}{k}-1} f(t) \mathrm{d} t, \quad x>a, k>0 .
$$

Note that

$$
\Gamma_{k}(x)=\int_{0}^{\infty} t^{x-1} e^{-\frac{t^{k}}{k}} \mathrm{~d} t
$$

is the $k$-Gamma function. Clearly

$$
\Gamma(x)=\lim _{k \rightarrow 1} \Gamma_{k}(x),
$$

and

$$
\Gamma_{k}(x+k)=x \Gamma_{k}(x) .
$$

For more details readers are referred to [16]. Note that when $k \rightarrow 1 k$-RiemannLiouville fractional integrals become classical Riemann-Liouville fractional integral [10]. $k$-Beta function is defined as:

$$
B_{k}(x, y)=\frac{1}{k} \int_{0}^{1} t^{\frac{x}{k}-1}(1-t)^{\frac{y}{k}-1} \mathrm{~d} t=\frac{\Gamma_{k}(x) \Gamma_{k}(y)}{\Gamma_{k}(x+y)}, \quad x>0, y>0 .
$$

Now, we prove Hermite-Hadmard inequality via $k$-Riemann-Liouville fractional integrals. 
Theorem 1. Let $f:[a, b] \rightarrow \mathbb{R}$ be positive function with $0 \leq a<b$ and $f \in L_{1}[a, b]$. If $f$ is convex on $[a, b]$, then, we have

$$
f\left(\frac{a+b}{2}\right) \leq \frac{\Gamma_{k}(\alpha+1)}{2(b-a)^{\frac{\alpha}{k}}}\left[{ }_{k} J_{a^{+}}^{\alpha} f(b)+{ }_{k} J_{b^{-}}^{\alpha} f(a)\right] \leq \frac{f(a)+f(b)}{2} .
$$

Proof. Since $f$ is convex function, so, we have

$$
f\left(\frac{x+y}{2}\right) \leq \frac{f(x)+f(y)}{2} .
$$

Let $x=t a+(1-t) b$ and $y=(1-t) a+t b$, we have

$$
2 f\left(\frac{a+b}{2}\right) \leq f(t a+(1-t) b)+f((1-t) a+t b) .
$$

Multiplying both sides of above inequality by $t^{\frac{\alpha}{k}-1}$ and then integrating with respect to $t$ on $[0,1]$, we have

$$
2 f\left(\frac{a+b}{2}\right) \int_{0}^{1} t^{\frac{\alpha}{k}-1} \mathrm{~d} t \leq \int_{0}^{1} t^{\frac{\alpha}{k}-1} f(t a+(1-t) b) \mathrm{d} t+\int_{0}^{1} t^{\frac{\alpha}{k}-1} f((1-t) a+t b) \mathrm{d} t .
$$

Now substituting $u=t a+(1-t) b$ and $v=(1-t) a+t b$, we have

$$
\frac{2 k}{\alpha} f\left(\frac{a+b}{2}\right) \leq \frac{k \Gamma_{k}(\alpha)}{(b-a)^{\frac{\alpha}{k}}}\left[{ }_{k} J_{a^{+}}^{\alpha} f(b)+{ }_{k} J_{b^{-}}^{\alpha} f(a)\right] .
$$

Also

$$
f(t a+(1-t) b)+f((1-t) a+t b) \leq f(a)+f(b)
$$

Multiplying both sides of above inequality $t^{\frac{\alpha}{k}-1}$ and then integrating with respect to $t$ on $[0,1]$, we have

$$
\frac{k \Gamma_{k}(\alpha)}{(b-a)^{\frac{\alpha}{k}}}\left[{ }_{k} J_{a^{+}}^{\alpha} f(b)+{ }_{k} J_{b^{-}}^{\alpha} f(a)\right] \leq \frac{k[f(a)+f(b)]}{\alpha} .
$$

Combining (2.4) and (2.5) completes the proof.

Note that when $k \rightarrow 1$ in Theorem 1, we have fractional Hermite-Hadamard inequality, see [17].

Lemma 1. Let $f:[a, b] \rightarrow \mathbb{R}$ be a differentiable mapping on $(a, b)$ with $a<b$. If $f \in L_{1}[a, b]$, then, we have

$$
\begin{aligned}
& \frac{b-a}{2} \int_{0}^{1}\left[t^{\frac{\alpha}{k}}-(1-t)^{\frac{\alpha}{k}}\right] f^{\prime}((1-t) a+t b) \mathrm{d} t \\
& =\frac{f(a)+f(b)}{2}-\frac{\Gamma_{k}(\alpha+1)}{2(b-a)^{\frac{\alpha}{k}}}\left[{ }_{k} J_{a^{+}}^{\alpha} f(a)+{ }_{k} J_{b^{-}}^{\alpha} f(b)\right] .
\end{aligned}
$$


Proof. Let

$$
I=\int_{0}^{1}\left[t^{\frac{\alpha}{k}}-(1-t)^{\frac{\alpha}{k}}\right] f^{\prime}((1-t) a+t b) \mathrm{d} t
$$

Now

$$
\begin{aligned}
& \int_{0}^{1} t^{\frac{\alpha}{k}} f^{\prime}((1-t) a+t b) \mathrm{d} t \\
& =\left[\frac{t^{\frac{\alpha}{k}} f((1-t) a+t b) \mathrm{d} t}{b-a}\right]_{0}^{1}-\frac{\alpha}{k(b-a)} \int_{0}^{1} t^{\frac{\alpha}{k}-1} f((1-t) a+t b) \mathrm{d} t \\
& =\frac{f(b)}{b-a}-\frac{k \alpha \Gamma_{k}(\alpha)}{k(b-a)^{\frac{\alpha}{k}+1} k \Gamma_{k}(\alpha)} \int_{a}^{b}(u-a)^{\frac{\alpha}{k}-1} f(u) \mathrm{d} u \\
& =\frac{f(b)}{b-a}-\frac{\Gamma_{k}(\alpha+1)}{(b-a)^{\frac{\alpha}{k}+1}}{ }_{k} J_{b^{-}}^{\alpha} f(a) .
\end{aligned}
$$

Similarly

$$
\int_{0}^{1}(1-t)^{\frac{\alpha}{k}} f^{\prime}((1-t) a+t b) \mathrm{d} t=-\frac{f(a)}{b-a}+\frac{\Gamma_{k}(\alpha+1)}{(b-a)^{\frac{\alpha}{k}+1}}{ }_{k} J_{a^{+}}^{\alpha} f(b) .
$$

Using (2.7) and (2.8) in (2.6) and then multiplying both sides by $\frac{b-a}{2}$, completes the proof.

Now using Lemma 1 we prove our next result, which plays a key role in the development of our next results.

Lemma 2. Let $f: I \rightarrow \mathbb{R}$ be twice differentiable function. If $f^{\prime \prime} \in L[a, b]$, then following equality for fractional integrals hold:

$$
\begin{aligned}
& \frac{f(a)+f(b)}{2}-\frac{\Gamma_{k}(\alpha+1)}{(b-a)^{\frac{\alpha}{k}}}\left[{ }_{k} J_{a^{+}}^{\alpha} f(a)+{ }_{k} J_{b^{-}}^{\alpha} f(b)\right] \\
& =\frac{k(b-a)^{2}}{2(\alpha+k)} \int_{0}^{1}\left[1-(1-t)^{\frac{\alpha}{k}+1}-t^{\frac{\alpha}{k}+1}\right] f^{\prime \prime}((1-t) a+t b) \mathrm{d} t .
\end{aligned}
$$

Proof. Now from Lemma 1, we have

$$
\int_{0}^{1}\left(t^{\frac{\alpha}{k}}-(1-t)^{\frac{\alpha}{k}}\right) f^{\prime}((1-t) a+t b) \mathrm{d} t
$$




$$
\begin{aligned}
= & k \int_{0}^{1} f^{\prime}((1-t) a+t b) d \frac{(1-t)^{\frac{\alpha}{k}+1}+t^{\frac{\alpha}{k}+1}}{\alpha+k} \\
=k & \left|\frac{(1-t)^{\frac{\alpha}{k}+1}+t^{\frac{\alpha}{k}+1}}{\alpha+k} f^{\prime}((1-t) a+t b)\right|_{0}^{1} \\
& \left.-(b-a) \int_{0}^{1} \frac{(1-t)^{\frac{\alpha}{k}+1}+t^{\frac{\alpha}{k}+1}}{\alpha+k} f^{\prime \prime}((1-t) a+t b) \mathrm{d} t\right\} \\
= & \frac{k}{\alpha+k}\left\{f^{\prime}(b)-f^{\prime}(a)-(b-a) \int_{0}^{1}\left[(1-t)^{\frac{\alpha}{k}+1}+t^{\frac{\alpha}{k}+1}\right] f^{\prime \prime}((1-t) a+t b) \mathrm{d} t\right\} .
\end{aligned}
$$

Also

$$
f^{\prime}(b)-f^{\prime}(a)=\int_{a}^{b} f^{\prime \prime}(x) \mathrm{d} x=(b-a) \int_{0}^{1} f^{\prime \prime}((1-t) a+t b) \mathrm{d} t .
$$

Utilizing (2.9), (2.10) and Lemma 1 completes the proof.

Note that when $k \rightarrow 1$ in Lemma 2, we get Lemma 2.1 [21].

\section{3. $k$-FRACTIONAL HERMiTE-HADAMARD INEQUALITIES VIA $s$-CONVEX FUNCTIONS OF FIRST SENSE}

In this section, we derive some $k$-fractional estimates of Hermite-Hadamard type inequalities via $s$-convex function of first sense.

Theorem 2. Let $f:[a, b] \rightarrow \mathbb{R}$ be twice differentiable on $(a, b)$ with $a<b$ and $f^{\prime \prime} \in L_{1}[a, b]$. If $\left|f^{\prime \prime}\right|$ is s-convex function in the first sense, then

$$
\begin{aligned}
& \left|\frac{f(a)+f(b)}{2}-\frac{\Gamma_{k}(\alpha+1)}{(b-a)^{\frac{\alpha}{k}}}\left[{ }_{k} J_{a^{+}}^{\alpha} f(a)+{ }_{k} J_{b^{-}}^{\alpha} f(b)\right]\right| \\
& \leq \frac{k(b-a)^{2}}{2(\alpha+k)}\left[\mathcal{G}_{1}(\alpha, k, s)\left|f^{\prime \prime}(a)\right|+\mathcal{G}_{2}(\alpha, k, s)\left|f^{\prime \prime}(b)\right|\right],
\end{aligned}
$$

where

$$
\begin{aligned}
& \mathcal{G}_{1}(\alpha, k, s)=\frac{s}{s+1}-\frac{2 k}{\alpha+2}+\frac{k}{\alpha+s k+2 k}+k B_{k}(k(s+1), \alpha+2 k) ; \\
& \mathcal{G}_{2}(\alpha, k, s)=\frac{1}{s+1}-\frac{k}{\alpha+s k+2 k}-k B_{k}(k(s+1), \alpha+2 k) .
\end{aligned}
$$


Proof. Using Lemma 2, property of the modulus and the fact that $\left|f^{\prime \prime}\right|$ is $s$-convex in the first sense, we have

$$
\begin{aligned}
& \left|\frac{f(a)+f(b)}{2}-\frac{\Gamma_{k}(\alpha+1)}{(b-a)^{\frac{\alpha}{k}}}\left[{ }_{k} J_{a^{+}}^{\alpha} f(a)+{ }_{k} J_{b^{-}}^{\alpha} f(b)\right]\right| \\
& \leq \frac{k(b-a)^{2}}{2(\alpha+k)} \int_{0}^{1}\left[1-(1-t)^{\frac{\alpha}{k}+1}-t^{\frac{\alpha}{k}+1}\right]\left|f^{\prime \prime}((1-t) a+t b)\right| \mathrm{d} t \\
& \leq \frac{k(b-a)^{2}}{2(\alpha+k)} \int_{0}^{1}\left[1-(1-t)^{\frac{\alpha}{k}+1}-t^{\frac{\alpha}{k}+1}\right]\left[\left(1-t^{s}\right)\left|f^{\prime \prime}(a)\right|+t^{s}\left|f^{\prime \prime}(b)\right|\right] \mathrm{d} t \\
& =\frac{k(b-a)^{2}}{2(\alpha+k)}\left[\left\{\frac{s}{s+1}-\frac{2 k}{\alpha+2}+\frac{k}{\alpha+s k+2 k}+k B_{k}(k(s+1), \alpha+2 k)\right\}\left|f^{\prime \prime}(a)\right|\right. \\
& \left.+\left\{\frac{1}{s+1}-\frac{k}{\alpha+s k+2 k}-k B_{k}(k(s+1), \alpha+2 k)\right\}\left|f^{\prime \prime}(b)\right|\right] .
\end{aligned}
$$

This completes the proof.

Theorem 3. Let $f:[a, b] \rightarrow \mathbb{R}$ be twice differentiable on $(a, b)$ with $a<b$ and $f^{\prime \prime} \in L_{1}[a, b]$. If $\left|f^{\prime \prime}\right|^{q}$ is s-convex function in the first sense, where $\frac{1}{p}+\frac{1}{q}=1, q>1$, then

$$
\begin{aligned}
& \left|\frac{f(a)+f(b)}{2}-\frac{\Gamma_{k}(\alpha+1)}{(b-a)^{\frac{\alpha}{k}}}\left[{ }_{k} J_{a^{+}}^{\alpha} f(a)+{ }_{k} J_{b^{-}}^{\alpha} f(b)\right]\right| \\
& \leq \frac{k(b-a)^{2}}{2(\alpha+k)}\left(\frac{p(\alpha+k)-k}{p(\alpha+k)+k}\right)^{\frac{1}{p}}\left(\frac{\left|f^{\prime \prime}(a)\right|^{q}+\left|f^{\prime \prime}(b)\right|^{q}}{s+1}\right)^{\frac{1}{q}} .
\end{aligned}
$$

Proof. Using Lemma 2, property of modulus, well-known Holder's inequality and the fact that $\left|f^{\prime \prime}\right|^{q}$ is $s$-convex in the first sense, we have

$$
\begin{aligned}
& \left|\frac{f(a)+f(b)}{2}-\frac{\Gamma_{k}(\alpha+1)}{(b-a)^{\frac{\alpha}{k}}}\left[{ }_{k} J_{a^{+}}^{\alpha} f(a)+{ }_{k} J_{b^{-}}^{\alpha} f(b)\right]\right| \\
& =\left|\frac{k(b-a)^{2}}{2(\alpha+k)} \int_{0}^{1}\left[1-(1-t)^{\frac{\alpha}{k}+1}-t^{\frac{\alpha}{k}+1}\right] f^{\prime \prime}((1-t) a+t b) \mathrm{d} t\right| \\
& \leq \frac{k(b-a)^{2}}{2(\alpha+k)}\left(\int_{0}^{1}\left(1-(1-t)^{\frac{\alpha}{k}+1}-t^{\frac{\alpha}{k}+1}\right)^{p} \mathrm{~d} t\right)^{\frac{1}{p}}\left(\int_{0}^{1}\left|f^{\prime \prime}((1-t) a+t b)\right|^{q} \mathrm{~d} t\right)^{\frac{1}{q}} \\
& \leq \frac{k(b-a)^{2}}{2(\alpha+k)}\left(\int_{0}^{1}\left(1-(1-t)^{p\left(\frac{\alpha}{k}+1\right)}-t^{p\left(\frac{\alpha}{k}+1\right)}\right) \mathrm{d} t\right)^{\frac{1}{p}}
\end{aligned}
$$




$$
\begin{gathered}
\times\left(\int_{0}^{1}\left[\left(1-t^{s}\right)\left|f^{\prime \prime}(a)\right|^{q}+t^{s}\left|f^{\prime \prime}(b)\right|^{q}\right] \mathrm{d} t\right)^{\frac{1}{q}} \\
=\frac{k(b-a)^{2}}{2(\alpha+k)}\left(\frac{p(\alpha+k)-k}{p(\alpha+k)+k}\right)^{\frac{1}{p}}\left(\frac{s\left|f^{\prime \prime}(a)\right|^{q}+\left|f^{\prime \prime}(b)\right|^{q}}{s+1}\right)^{\frac{1}{q}} .
\end{gathered}
$$

This completes the proof.

Theorem 4. Let $f:[a, b] \rightarrow \mathbb{R}$ be twice differentiable on $(a, b)$ with $a<b$ and $f^{\prime \prime} \in L_{1}[a, b]$. If $\left|f^{\prime \prime}\right|^{q}$ is s-convex function in the first sense, where $q>1$, then

$$
\begin{aligned}
& \left|\frac{f(a)+f(b)}{2}-\frac{\Gamma_{k}(\alpha+1)}{(b-a)^{\frac{\alpha}{k}}}\left[{ }_{k} J_{a^{+}}^{\alpha} f(a)+{ }_{k} J_{b^{-}}^{\alpha} f(b)\right]\right| \\
& \leq \frac{k(b-a)^{2}}{2(\alpha+k)}\left(\frac{\alpha+2(k-1)}{\alpha+2 k}\right)^{1-\frac{1}{q}}\left(\mathcal{G}_{1}(\alpha, k, s)\left|f^{\prime \prime}(a)\right|^{q}+\mathcal{G}_{2}(\alpha, k, s)\left|f^{\prime \prime}(b)\right|^{q}\right)^{\frac{1}{q}},
\end{aligned}
$$

where $\mathcal{G}_{1}(\alpha, k, s)$ and $\mathcal{G}_{2}(\alpha, k, s)$ are given by (3.1) and (3.2) respectively.

Proof. Using Lemma 2, property of modulus, well-known power-mean inequality and the fact that $\left|f^{\prime \prime}\right|^{q}$ is $s$-convex in the first sense, we have

$$
\begin{aligned}
\left|\frac{f(a)+f(b)}{2}-\frac{\Gamma_{k}(\alpha+1)}{(b-a)^{\frac{\alpha}{k}}}\left[{ }_{k} J_{a^{+}}^{\alpha} f(a)+{ }_{k} J_{b^{-}}^{\alpha} f(b)\right]\right| \\
=\left|\frac{k(b-a)^{2}}{2(\alpha+k)} \int_{0}^{1}\left[1-(1-t)^{\frac{\alpha}{k}+1}-t^{\frac{\alpha}{k}+1}\right] f^{\prime \prime}((1-t) a+t b) \mathrm{d} t\right| \\
\leq \frac{k(b-a)^{2}}{2(\alpha+k)}\left(\int_{0}^{1}\left[1-(1-t)^{\frac{\alpha}{k}+1}-t^{\frac{\alpha}{k}+1}\right] \mathrm{d} t\right)^{1-\frac{1}{q}} \\
\quad \times\left(\int_{0}^{1}\left(1-(1-t)^{\frac{\alpha}{k}+1}-t^{\frac{\alpha}{k}+1}\right)\left|f^{\prime \prime}((1-t) a+t b)\right|^{q} \mathrm{~d} t\right)^{\frac{1}{q}} \\
\leq \frac{k(b-a)^{2}}{2(\alpha+k)}\left(\frac{\alpha+2(k-1)}{\alpha+2 k}\right)^{1-\frac{1}{q}}\left(\mathcal{G}_{1}(\alpha, k, s)\left|f^{\prime \prime}(a)\right|^{q}+\mathcal{G}_{2}(\alpha, k, s)\left|f^{\prime \prime}(b)\right|^{q}\right)^{\frac{1}{q}} .
\end{aligned}
$$

This completes the proof.

\section{4. $k$-FRACTIONAL HERMITE-HADAMARD INEQUALITIES VIA $s$-CONVEX FUNCTIONS OF SECOND SENSE}

In this section, we derive some $k$-fractional estimates of Hermite-Hadamard type inequalities via $s$-convex function of second sense. 
Theorem 5. Let $f:[a, b] \rightarrow \mathbb{R}$ be twice differentiable on $(a, b)$ with $a<b$ and $f^{\prime \prime} \in L_{1}[a, b]$. If $\left|f^{\prime \prime}\right|$ is s-convex function in the second sense, then

$$
\begin{aligned}
& \left|\frac{f(a)+f(b)}{2}-\frac{\Gamma_{k}(\alpha+1)}{(b-a)^{\frac{\alpha}{k}}}\left[{ }_{k} J_{a^{+}}^{\alpha} f(a)+{ }_{k} J_{b^{-}}^{\alpha} f(b)\right]\right| \\
& \leq \frac{k(b-a)^{2}}{2(\alpha+k)} \mathcal{H}(\alpha, k, s)\left[\left|f^{\prime \prime}(a)\right|+\left|f^{\prime \prime}(b)\right|\right],
\end{aligned}
$$

where

$$
\mathcal{H}(\alpha, k, s)=\frac{1}{s+1}-\frac{k}{\alpha+s k+2 k}-k B_{k}(\alpha+2 k, k(s+1)) .
$$

Proof. Using Lemma 2, property of modulus and the fact that $\left|f^{\prime \prime}\right|$ is $s$-convex in the second sense, we have

$$
\begin{aligned}
& \left|\frac{f(a)+f(b)}{2}-\frac{\Gamma_{k}(\alpha+1)}{(b-a)^{\frac{\alpha}{k}}}\left[{ }_{k} J_{a^{+}}^{\alpha} f(a)+{ }_{k} J_{b^{-}}^{\alpha} f(b)\right]\right| \\
& \leq \frac{k(b-a)^{2}}{2(\alpha+k)} \int_{0}^{1}\left[1-(1-t)^{\frac{\alpha}{k}+1}-t^{\frac{\alpha}{k}+1}\right]\left|f^{\prime \prime}((1-t) a+t b)\right| \mathrm{d} t \\
& \leq \frac{k(b-a)^{2}}{2(\alpha+k)} \int_{0}^{1}\left[1-(1-t)^{\frac{\alpha}{k}+1}-t^{\frac{\alpha}{k}+1}\right]\left[(1-t)^{s}\left|f^{\prime \prime}(a)\right|+t^{s}\left|f^{\prime \prime}(b)\right|\right] \mathrm{d} t \\
& =\frac{k(b-a)^{2}}{2(\alpha+k)}\left[\frac{1}{s+1}-\frac{k}{\alpha+s k+2 k}-k B_{k}(\alpha+2 k, k(s+1))\right]\left[\left|f^{\prime \prime}(a)\right|+\left|f^{\prime \prime}(b)\right|\right] .
\end{aligned}
$$

This completes the proof.

Theorem 6. Let $f:[a, b] \rightarrow \mathbb{R}$ be twice differentiable on $(a, b)$ with $a<b$ and $f^{\prime \prime} \in L_{1}[a, b]$. If $\left|f^{\prime \prime}\right|^{q}$ is s-convex function in the second sense, where $\frac{1}{p}+\frac{1}{q}=1$, $q>1$, then

$$
\begin{aligned}
& \left|\frac{f(a)+f(b)}{2}-\frac{\Gamma_{k}(\alpha+1)}{(b-a)^{\frac{\alpha}{k}}}\left[{ }_{k} J_{a^{+}}^{\alpha} f(a)+{ }_{k} J_{b^{-}}^{\alpha} f(b)\right]\right| \\
& \leq \frac{k(b-a)^{2}}{2(\alpha+k)}\left(\frac{p(\alpha+k)-k}{p(\alpha+k)+k}\right)^{\frac{1}{p}}\left(\frac{\left|f^{\prime \prime}(a)\right|^{q}+\left|f^{\prime \prime}(b)\right|^{q}}{s+1}\right)^{\frac{1}{q}} .
\end{aligned}
$$

Proof. Using Lemma 2, property of modulus, well-known Holder's inequality and the fact that $\left|f^{\prime \prime}\right|^{q}$ is $s$-convex in the second sense, we have

$$
\begin{aligned}
& \left|\frac{f(a)+f(b)}{2}-\frac{\Gamma_{k}(\alpha+1)}{(b-a)^{\frac{\alpha}{k}}}\left[{ }_{k} J_{a^{+}}^{\alpha} f(a)+{ }_{k} J_{b^{-}}^{\alpha} f(b)\right]\right| \\
& =\left|\frac{k(b-a)^{2}}{2(\alpha+k)} \int_{0}^{1}\left[1-(1-t)^{\frac{\alpha}{k}+1}-t^{\frac{\alpha}{k}+1}\right] f^{\prime \prime}((1-t) a+t b) \mathrm{d} t\right|
\end{aligned}
$$




$$
\begin{gathered}
\leq \frac{k(b-a)^{2}}{2(\alpha+k)}\left(\int_{0}^{1}\left(1-(1-t)^{\frac{\alpha}{k}+1}-t^{\frac{\alpha}{k}+1}\right)^{p} \mathrm{~d} t\right)^{\frac{1}{p}}\left(\int_{0}^{1}\left|f^{\prime \prime}((1-t) a+t b)\right|^{q} \mathrm{~d} t\right)^{\frac{1}{q}} \\
\leq \frac{k(b-a)^{2}}{2(\alpha+k)}\left(\int_{0}^{1}\left(1-(1-t)^{p\left(\frac{\alpha}{k}+1\right)}-t^{p\left(\frac{\alpha}{k}+1\right)}\right) \mathrm{d} t\right)^{\frac{1}{p}} \\
\times\left(\int_{0}^{1}\left[(1-t)^{s}\left|f^{\prime \prime}(a)\right|^{q}+t^{s}\left|f^{\prime \prime}(b)\right|^{q}\right] \mathrm{d} t\right)^{\frac{1}{q}} \\
=\frac{k(b-a)^{2}}{2(\alpha+k)}\left(\frac{p(\alpha+k)-k}{p(\alpha+k)+k}\right)^{\frac{1}{p}}\left(\frac{\left|f^{\prime \prime}(a)\right|^{q}+\left|f^{\prime \prime}(b)\right|^{q}}{s+1}\right)^{\frac{1}{q}} \cdot
\end{gathered}
$$

This completes the proof.

Theorem 7. Let $f:[a, b] \rightarrow \mathbb{R}$ be twice differentiable on $(a, b)$ with $a<b$ and $f^{\prime \prime} \in L_{1}[a, b]$. If $\left|f^{\prime \prime}\right|^{q}$ is s-convex function in the second sense where $q>1$, then

$$
\begin{aligned}
& \left|\frac{f(a)+f(b)}{2}-\frac{\Gamma_{k}(\alpha+1)}{(b-a)^{\frac{\alpha}{k}}}\left[{ }_{k} J_{a^{+}}^{\alpha} f(a)+{ }_{k} J_{b^{-}}^{\alpha} f(b)\right]\right| \\
& \leq \frac{k(b-a)^{2}}{2(\alpha+k)} \mathcal{H}^{\frac{1}{q}}(\alpha, k, s)\left[\left|f^{\prime \prime}(a)\right|^{q}+\left|f^{\prime \prime}(b)\right|^{q}\right]^{\frac{1}{q}},
\end{aligned}
$$

where $\mathcal{H}(\alpha, k, s)$ is given by (4.1).

Proof. Using Lemma 2, property of modulus, well-known power-mean inequality and the fact that $\left|f^{\prime \prime}\right|^{q}$ is $s$-convex in the second sense, we have

$$
\begin{aligned}
& \left|\frac{f(a)+f(b)}{2}-\frac{\Gamma_{k}(\alpha+1)}{(b-a)^{\frac{\alpha}{k}}}\left[{ }_{k} J_{a^{+}}^{\alpha} f(a)+{ }_{k} J_{b^{-}}^{\alpha} f(b)\right]\right| \\
& =\left|\frac{k(b-a)^{2}}{2(\alpha+k)} \int_{0}^{1}\left[1-(1-t)^{\frac{\alpha}{k}+1}-t^{\frac{\alpha}{k}+1}\right] f^{\prime \prime}((1-t) a+t b) \mathrm{d} t\right| \\
& \leq \frac{k(b-a)^{2}}{2(\alpha+k)}\left(\int_{0}^{1}\left[1-(1-t)^{\frac{\alpha}{k}+1}-t^{\frac{\alpha}{k}+1}\right] \mathrm{d} t\right)^{1-\frac{1}{q}} \\
& \quad \times\left(\int_{0}^{1}\left(1-(1-t)^{\frac{\alpha}{k}+1}-t^{\frac{\alpha}{k}+1}\right)\left|f^{\prime \prime}((1-t) a+t b)\right|^{q} \mathrm{~d} t\right)^{\frac{1}{q}}
\end{aligned}
$$




$$
\begin{aligned}
\leq & \frac{k(b-a)^{2}}{2(\alpha+k)}\left(\frac{\alpha+2(k-1)}{\alpha+2 k}\right)^{1-\frac{1}{q}} \\
& \left.\times\left(\frac{1}{s+1}-\frac{k}{\alpha+s k+2 k}-k B_{k}(\alpha+2 k, k(s+1))\right)^{\frac{1}{q}}\left(\left|f^{\prime \prime}(a)\right|^{q}+\left|f^{\prime \prime}(b)\right|^{q}\right]\right)^{\frac{1}{q}} \\
= & \left.\frac{(b-a)^{2}}{2(\alpha+1)}\left(\frac{\alpha}{\alpha+2}\right)^{1-\frac{1}{q}} \mathcal{H}^{\frac{1}{q}}(\alpha, k, s)\left(\left|f^{\prime \prime}(a)\right|^{q}+\left|f^{\prime \prime}(b)\right|^{q}\right]\right)^{\frac{1}{q}} .
\end{aligned}
$$

This completes the proof.

Remark 1 . We would like to point out that for $s=1$ results obtained in section 3 and 4 reduces to the result for classical convexity. The results for classical convexity also appear to be new in the literature.

\section{ACKNOWLEDGEMENT}

Authors are thankful to anonymous referee for his/her valuable comments and suggestions. Authors are also grateful to Dr. S. M. Junaid Zaidi, Rector, COMSATS Institute of Information Technology, Pakistan for providing excellent research facilities.

\section{REFERENCES}

[1] W. W. Breckner, "Stetigkeitsaussagen für eine Klasse verallgemeinerter konvexer Funktionen in topologischen linearen Räumen,” Publ. Inst. Math., Nouv. Sér., vol. 23, pp. 13-20, 1978.

[2] P. Burai, A. Házy, and T. Juhász, "Bernstein-Doetsch type results for $s$-convex functions," Publ. Math., vol. 75, no. 1-2, pp. 23-31, 2009.

[3] P. Burai, A. Házy, and T. Juhász, "On approximately Breckner s-convex functions," Control Cybern., vol. 40, no. 1, pp. 91-99, 2011.

[4] G. Cristescu, M. Aslam Noor, and M. Uzair Awan, "Bounds of the second degree cumulative frontier gaps of functions with generalized convexity," Carpathian J. Math., vol. 31, no. 2, pp. 173-180, 2015.

[5] G. Cristescu and L. Lupşa, Non-connected convexities and applications. Dordrecht: Kluwer Academic Publishers, 2002, vol. 68.

[6] G. Cristescu, M. A. Noor, K. I. Noor, and M. U. Awan, "Some inequalities for functions having Orlicz-convexity," Appl. Math. Comput., vol. 273, pp. 226-236, 2016.

[7] S. S. Dragomir and C. E. M. Pearce, Selected topics on Hermite-Hadamard inequalities and applications. Victoria University, Australia, 2000.

[8] S. S. Dragomir, J. E. Pečarić, and L. E. Persson, "Some inequalities of Hadamard type," Soochow J. Math., vol. 21, no. 3, pp. 335-341, 1995.

[9] A. Házy, "Bernstein-Doetsch type results for $(k, h)$-convex functions," Miskolc Math. Notes, vol. 13, no. 2, pp. 325-336, 2012.

[10] A. A. Kilbas, H. M. Srivastava, and J. J. Trujillo, Theory and applications of fractional differential equations. Amsterdam: Elsevier, 2006, vol. 204.

[11] M. V. Mihai, M. A. Noor, K. I. Noor, and M. U. Awan, "Some integral inequalities for harmonic h-convex functions involving hypergeometric functions," Appl. Math. Comput., vol. 252, pp. 257$262,2015$. 
[12] M. A. Noor and M. U. Awan, "Some Integral inequalities for two kinds of convexities via fractional integrals," Trans. J. Math. Mech., vol. 5, no. 2, pp. 129-136, 2013.

[13] M. A. Noor, K. I. Noor, and M. U. Awan, "Fractional Hermite-Hadmard inequalities for convex functions and applications," Tbilisi Mathematical Journal, vol. 8, no. 2, pp. 103-113, 2015.

[14] M. A. Noor, G. Cristescu, and M. U. Awan, "Generalized fractional Hermite-Hadamard inequalities for twice differentiable $s$-convex functions," Filomat, vol. 29, no. 4, pp. 807-815, 2015.

[15] M. E. Ozdemir, C. Yildiz, and H. Kavurmaci, "Fractional integral inequalities for different functions," arXiv:1202.2431v1, 2012.

[16] M. Z. Sarikaya and A. Karaca, "On the $k$-Riemann-Liouville fractional integral and applications," Int. J. Stat. Math., vol. 1, no. 3, pp. 33-43, 2014.

[17] M. Z. Sarikaya, E. Set, H. Yaldiz, and N. Başak, "Hermite-Hadamard's inequalities for fractional integrals and related fractional inequalities," Math. Comput. Modelling, vol. 57, no. 9-10, pp. 2403-2407, 2013.

[18] G. Toader, "The hierarchy of convexity and some classic inequalities," J. Math. Inequal., vol. 3, no. 3, pp. 305-313, 2009 .

[19] M. Tunç, "On new inequalities for $h$-convex functions via Riemann-Liouville fractional integration," Filomat, vol. 27, no. 4, pp. 559-565, 2013.

[20] M. Tunç, "On some integral inequalities via $h$-convexity," Miskolc Math. Notes, vol. 14, no. 3, pp. 1041-1057, 2013.

[21] J. R. Wang, X. Li, M. Fečkan, and Y. Zhou, "Hermite-Hadamard-type inequalities for RiemannLiouville fractional integrals via two kinds of convexity," Appl. Anal., vol. 92, no. 11, pp. 22412253, 2013.

[22] J. Wang, X. Li, and C. Zhu, "Refinements of Hermite-Hadamard type inequalities involving fractional integrals," Bull. Belg. Math. Soc. - Simon Stevin, vol. 20, no. 4, pp. 655-666, 2013.

[23] J. Wang, C. Zhu, and Y. Zhou, "New generalized Hermite-Hadamard type inequalities and applications to special means," J. Inequal. Appl., vol. 2013, p. 15, 2013, id/No 325.

[24] B.-Y. Xi and F. Qi, "Some Hermite-Hadamard type inequalities for differentiable convex functions and applications," Hacet. J. Math. Stat., vol. 42, no. 3, pp. 243-257, 2013.

Authors' addresses

Muhammad Aslam Noor

Department of Mathematics, COMSATS Institute of Information Technology, Park Road, Islamabad, Pakistan

E-mail address: noormaslamegmail.com

Khalida Inayat Noor

Department of Mathematics, COMSATS Institute of Information Technology, Park Road, Islamabad, Pakistan

E-mail address: khalidanoor@hotmail.com

Muhammad Uzair Awan

(corresponding author) Department of Mathematics, COMSATS Institute of Information Technology, Park Road, Islamabad, Pakistan

E-mail address: awan.uzair@gmail.com 